Bahar et al., Afr J Tradit Complement Altern Med. (2013) 10(2):292-298 292

http://dx.doi.org/10.4314/ajtcam.v10i2.14

\title{
HERBAL THERAPIES USED BY HYPERTENSIVE PATIENTS IN TURKEY
}

\author{
Zuhal Bahar, ${ }^{1}$ Sevgi Kızılcı, Ayşe Beşer, ${ }^{1}$ Dilek Büyükkaya Besen, Nihal Gördes, Fatma \\ Ersin, Aygul Kissal, ${ }^{2}$ Cantürk Çapık
}

\author{
University of Nursing Faculty, Public Health Nursing Department, Inciralti, Izmir, Turkey \\ ${ }^{1}$ University of Nursing Faculty, Internal Medicine Nursing Department, Inciralti, Izmir, Turkey \\ Telephone: +90 23241247 77; Fax: +90 23241247 98; e-mail: sevgi.kizilci@deu.edu.tr \\ ${ }^{2}$ Faculty of Health, Kars, Turkey \\ *E-mail: zuhal.bahar@deu.edu.tr
}

\begin{abstract}
The purpose of this study was to determine herbal therapies used by the patients for regulation of blood pressure. This descriptive study was conducted in three districts of Izmir, Turkey. The study group included 193 patients with hypertension diagnosis who admitted to a primary care center and accepted to participate in the study. Data were collected through an investigator-made questionnaire including questions about socio-demographic features and herbal therapies used for regulation of blood pressure. Percentiles and chi-square statistics tests were used to evaluate the data through SPSS 11.0 program. The subjects included in the study had suffered from hypertension for $8.28 \pm 7.92$ years, and $95.9 \%$ of them received medication to treat their hypertension. Of all hypertensive patients, $51.3 \%$ used herbal therapies for the treatment of hypertension. The patients used herbal therapies most commonly when they felt ill. There were no statistically significant difference between the use of herbal therapies and gender, education, marital status, having health insurance and income level. Health professionals who provide primary health services, especially nurses, need to follow patients regularly and provide patient education on antihypertensive therapy. Nurses must plan the necessary training programs by evaluating the data about, whether the hypertension patients use herbal therapies that can affect their health negatively or not.
\end{abstract}

Key words: Hypertension, Therapies, Herbalism, Nursing Care.

\section{Introduction}

Hypertension is a major health problem in most countries. The worldwide prevalence of hypertension is $25-30 \%$ in the adult population and reaches $60-70 \%$ in people aged over 60 years (Vasan et al., 2002). There are about 50 million hypertensive patients in the United States and more than half of them are aged 60 years or over (National-Heart-Lung-andBlood-Institute, 2008). Due to insufficient hypertension prevention and control at the community level, hypertension results in increased mortality and morbidity. Fields et al. (2004) reported that hypertension affects nearly one billion individuals worldwide, with expected increases as the average life expectancy rises. According to the data of World Health Organization (WHO, 2005a) hypertension accounts for more than $5.8 \%$ of total deaths, $1.9 \%$ of years of life lost and $1.4 \%$ of disability adjusted life years all over the world.

At present, blood pressure can be controlled through medical treatment and healthy life- style. However, studies suggest that alternative treatment options are often used in addition to medical treatment. It has been reported in the world literature that green tea, polyphenol rich dark chocolate grapes, ginger, thyme, cinnamon, ginseng, mint and grapefruit are also used to prevent hypertension (Buettner, Phillips, Davis, Gardiner, \& Mittleman, 2007; Nahas, 2008; Wangzhong, 1996). Several studies have focused on the effects of herbal therapies and CAM (complementary and alternative medicine) including nutrients, garlic, exercise, relaxation methods and massage. Garlic affects the rennin-angiotensin system and nitric oxide and thus reduces blood pressure (Al-Qattan et al., 2006). It has been claimed that grape juice increases the synthesis of nitric oxide, which protects vessels (Fantinelli \& Mosca, 2007; Jimenez et al., 2007) and that lemon juice, parsley leaves and roots have a diuretic effect (Castleman, 2005). Duffy et al. (1999) reported that vitamin C was used to decrease systolic blood pressure. In essential hypertensive patients, impaired endothelial vasodilation can be improved by the antioxidant vitamin C (Taddei et al., 1998). Preuss, Echard, Polansky, and Anderson (2006) noted that cinnamon lowered systolic blood pressure. Studies on yogurt, the main ingredient of airan (a drink made of yogurt, salt and water) have revealed that yogurt decreases blood pressure, which has been attributed to the calcium (Martinez, 1998; Preuss, et al., 2006).

When analyzed properly, complementary and alternative therapies used by hypertensive patients might be useful to promote understanding of self-care regimen and health behaviors of the hypertensive patients. There are several complementary and alternative therapies such as mind-body intervention, bio-electromagnetic therapies, alternative system of medical practice, manual healing methods, biological treatment, herbal medicine, diet and nutritional therapies. This study only focuses on herbal therapy because herbal therapy is used more, by comparison with the other treatments administered in Turkey.

\section{Prevalence of Hypertension and Hypertension Related Herbal Therapies in Turkey}

In Turkey, the prevalence of hypertension is $31.8 \%$ in adults over the age of 18 years, and reaches $75.1 \%$ in individuals that are 65 years old and over. In older adults, it is more common in women $(81.7 \%)$ than men $(67.2 \%)$ 
(Kirkpantur \& Turgan, 2006). The rate of blood pressure under control is 8.1\% (Kurkpantur \& Turgan, 2006). Hypertension is an important cause of both morbidity and mortality and is considered as a serious public health problem. There have been attempts to prevent and diagnose hypertension earlier in primary health care facilities. Patients diagnosed as hypertansive get care from primary health centers whenever they apply. However, they are not invited to follow up routinely; therefore, there is no special separate records. Nurses in the primary health care centers offer hypertansive patients education on various subjects such as health care, nutrition, exercise and weigh management in addition to medical treatment of hypertension by physicians. Cases of hypertension which are not possible to be treated in primary care facilities need hospital care. In Turkey, not only health care facilities but also traditional practices play an important role in fulfilling health care needs. Patients with chronic diseases such as hypertension can consider herbal therapies as an alternative solution to management of their diseases and they may interact with each other.

Akpinar and Tezel (2003) found that $86.9 \%$ of hypertensive patient in Turkey used treatment alternatives, such as garlic $(44.3 \%)$, lemon juice $(42.5 \%)$, airan (31\%) and a special liquid derived from boiled olive tree leaves $(23.9 \%)$. Toprak and Demir (2007) suggests that, when their blood pressures rises hypertensive patients consume yogurt with garlic $(27.8 \%)$, sour foods such as lemon and grapefruit (25.8\%), airan (5.6\%), and/or garden thyme juice $(2.8 \%)$.

The overall effectiveness of traditional, CAM on managing high blood pressure is not clear. In conflict with the studies mentioned above, several studies suggest harmful effects of herbal therapies (Dosh, 2002; Duffy et al., 1999; Edwards, Colquist, \& Maradiegue, 2005; Goertz, Grimm, Svendsen, \& Grandits, 2002; He \& MacGregor, 2002; Mansoor, 2001). For example, when combined with anticoagulant, anti-platelet and non-steroidal anti- inflammatory drugs, garlic has been reported to increase the risk of bleeding (Edwards et al., 2005; Hu et al., 2005). In addition, garlic may cause stomach upset, nausea, vomiting, diarrhea, contact dermatitis, bad breath and an unpleasant skin odor (Edwards et al., 2005; Hu et al., 2005). Some other plants may also increase blood pressure. For example, licorice roots have been reported to cause hypertension when used in high doses (Dharmananda, 2003).

A few studies have been conducted in Turkey to explore health behaviours and herbal therapies used to reduce blood pressure (Akpınar \& Tezel, 2003; Toprak \& Demir, 2007). However, these studies have not been able to show a complete profile of the herbal therapies used. Therefore, further studies on health behaviours and herbal therapies are needed. This research will help nurses, midwives and physicians responsible for primary health care services to develop appropriate health promotion and counseling programs that assist hypertensive patients to adopt appropriate health behaviors.

The aim of this study was to identify herbal therapies used by hypertensive patients to reduce their blood pressure. Specific research questions include:

-What are the most frequent herbal therapies used by hypertensive patients?

-Is there a difference between the gender of hypertensive patients and use of herbal therapies?

-Is there a difference between the education level of hypertensive patients and use of herbal therapies?

-Is there a difference between the marital status of hypertensive patients and use of herbal therapies?

-Is there a difference between the income of hypertensive patients and use of herbal therapies?

\section{Method \\ Setting}

The study was conducted in the primary care centers in Esentepe, Yelki and Güzelbahçe districts of Izmir, located in the western part of Turkey. Yelki is a rural area where people make their living from agriculture and livestock. In general, Yelki is made up of individuals with low socio-economic status. By contrast, Güzelbahçe is composed of individuals with a high socio-economic status. People in Güzelbahçe make their living from fishing and other economic sectors. Esentepe is an urban area where people migrate from eastern and central parts of Turkey. Inhabitants with low, moderate and high level of income live in Esentepe. These three districts were selected because of their different levels of income.

\section{Sample and Sampling Procedures}

Even though all of the individuals who reside in the region are registered in health centers, there is no data showing that they have hypertension. Therefore, we asked local authorities to make announcements to invite hypertensive patients to come to the health centers. The Municipalities (local authorities) announced that "hypertensive individuals can admit to primary care centers for blood pressure measurements and to get consultation services" in order to reach the patients. Also, the patients whose phone numbers were available were called and invited to the health centers. After the announcements 193 patients with hypertension diagnosis were admitted to primary care center. The study sample included 75 patients from Güzelbahçe, 65 patients from Yelki and 53 patients from Esentepe. The patients whose blood pressure was measured were then informed about the study and its aim and asked whether they would participate. All the patients accepted to take part in the study. They were assured that the obtained data would be kept confidential. The study was explained to potential study subjects by one of the research investigators.

The patients were asked for consent orally.

\section{Data Collection Method}

To obtain reliable information, data were collected through face-to-face interviews in a room provided at the primary health centers for the study and the answers of the patients were recorded by the researchers themselves. The interviews took about 20 minutes. 


\section{Data Collection Tools}

The data collection tool was developed by the investigators based on the information derived from relevant literature. After the researcher developed the instrument, two internal disease specialists and two public health specialists in nursing were asked to comment on the questions included in the questionnaire. The questionnaire was revised in accordance with the comments made by the specialists. Additionally, before data collection, in order to determine whether the questions were understandable, the questionnaire was distributed to ten hypertensive patients that were not included in the study sample. Modifications were made where necessary. The questionnaire included 24 open-ended and yes/no questions about socio demographic features, herbal therapies used to lower blood pressure, alternative treatment methods used to treat conditions other than hypertension and health behavior. The open ended questions that were asked included:

-Do you use any methods other than medical treatment/drugs to keep your blood pressure under control?

-What do you take instead of or in addition to medical treatment/drugs to lower your blood pressure?

-How do you prepare them?

-How are they used?

-What is the source of your knowledge about the methods you use instead of or together with medical treatment/drugs to lower your blood pressure?

\section{Inclusion and Exclusion Criteria}

The inclusion criteria were being diagnosed with hypertension by a physician, receiving treatment for hypertension and the ability to communicate effectively in Turkish. Exclusion criteria were hearing and perception problems. Because the study was examining herbal therapies specifically used for hypertension, individuals with another illness in addition to hypertension were also excluded from the study.

\section{Ethical Consideration}

Prior to conducting the research, Ethics Committee of the School of Nursing at Dokuz Eylul University was asked for approval. Ethical approval was also given by the local Health Department in Izmir.

\section{Data Analysis}

SPSS 11 software was used for data analysis. The participants who used herbal therapies were coded as "yes" and those who did not use herbal therapies were coded as "no". The Chi-square statistics test was used to determine whether there was a difference between the use of herbal therapies for the treatment of hypertension and their age, gender, education, marital status, income, health insurance and the districts of residence.

\section{Results}

The mean age of the study sample was $61.04 \pm 11.89$ years. Seventy-two percent of the subjects were female. In terms of education, $53.9 \%$ were primary school graduates, $15 \%$ were illiterate, $13.5 \%$ were literate and $17.6 \%$ were high school and university graduates. (In Turkey, the term "literate" is used to refer to people who learn reading and writing in training programs organized by the government or non govermental organizations in public education centers rather than in schools of the Ministry of Education). Eighty-five percent of the subjects were married, $93.8 \%$ did not have any health insurance and $43 \%$ had an income that was lower than their expenditures.

The subjects included in the study had suffered from hypertension for $8.28+7.92$ years, and $95.9 \%$ of them had received medication to treat their hypertension. The latter group of patients did not receive treatment because $44.4 \%$ of them had been newly diagnosed, $22.2 \%$ thought that they did not need treatment and $11.1 \%$ could not afford their prescription. Of all hypertensive subjects, $51.3 \%$ used herbal therapies for the treatment of hypertension.

Table 1 shows the most common herbal therapies used to control or treat hypertension. The herbal therapies used by the patients $(\mathrm{n}=99)$ included lemon juice $(36.4 \%)$ and a mixture of lemon juice and garlic $(27.3 \%)$. When examining preparation methods, it was reported that nar eksisi (sour pomegranate juice), grape juice, and lemon alone or with water were drunk, garlic and flax seeds were chewed in their natural state or swallowed whole, and olive tree leaves, thyme, mint, and lavender flowers were boiled in water and then drunk. The patients used herbal therapies most commonly when they felt ill. Most often, they learned about herbal therapies from their neighbors. The patients used only herbal therapies but they did not use any other methods such as mind-body intervention, bio-electromagnetic therapies, alternative system of medical practice etc).

As shown in Table 2, there were no statistically significant differences between the use of herbal therapies practice and gender, district, education, marital status, health insurance or income.

\section{Discussion}

Most of the herbal therapies used by the participants of this study are similar to those reported from other studies in the literature. Airan, garlic, parsley, cinnamon, lemon and flax seeds are natural foods recommended for healthy nutrition. However, they may be harmful when consumed in higher amounts and they may interact with drugs used for hypertension 
Bahar et al., Afr J Tradit Complement Altern Med. (2013) 10(2):292-298 295 http://dx.doi.org/10.4314/ajtcam.v10i2.14

Table 1: Characteristics of the Traditional Methods Used by Hypertensive Patients $(n=99)$

\begin{tabular}{|c|c|c|c|}
\hline & & $\mathbf{n}$ & $\%$ \\
\hline \multirow{14}{*}{$\begin{array}{l}\text { Traditional Therapies used } \\
\text { by Hypertensive Patients } \\
(\mathrm{n}=99)\end{array}$} & Lemon juice & 36 & 33.6 \\
\hline & A mixture of lemon and garlic & 27 & 25.2 \\
\hline & Garlic & 16 & 15.0 \\
\hline & A mixture of lemon and parsley/lavender flowers /sour & 15 & 14.0 \\
\hline & pomegranate mixture /airan/ soda/ olive oil tree leaves & & \\
\hline & Flax seed & 2 & 1.9 \\
\hline & Thyme & 4 & 3.7 \\
\hline & Airan without salt & 2 & 1.9 \\
\hline & Yoghurt with garlic & 1 & 0.9 \\
\hline & Green tea & 1 & 0.9 \\
\hline & Apple wine & 1 & 0.9 \\
\hline & Cinnamon & 1 & 0.9 \\
\hline & Laurel leaves & 1 & 0.9 \\
\hline & Total* & $107^{*}$ & 100.0 \\
\hline \multirow{4}{*}{$\begin{array}{l}\text { How do you prepare the } \\
\text { therapy? } \\
\text { (n=96; } 3 \text { individuals did } \\
\text { not answer.) }\end{array}$} & $\begin{array}{l}\text { Drunk as is or after mixing with water (sour pomegranate } \\
\text { mixture, grape juice, lemon juice) }\end{array}$ & 48 & 50.0 \\
\hline & Chewed in natural state or swallowed whole (garlic, flax seed) & 41 & 42.7 \\
\hline & $\begin{array}{l}\text { Boiled with water and drunk (olive tree leaves, thyme, mint, } \\
\text { lavender flower) }\end{array}$ & 7 & 7.3 \\
\hline & Total & 96 & 100 \\
\hline \multirow{4}{*}{$\begin{array}{l}\text { How often are they used? } \\
\text { ( } \mathrm{n}=88 ; 11 \text { individuals did } \\
\text { not answer) }\end{array}$} & When their blood pressure increases or they feel ill & 43 & 48.9 \\
\hline & Every day (before breakfast or in the evening) & 32 & 36.3 \\
\hline & Occasionally/when remembered & 13 & 14.8 \\
\hline & Total & 88 & 100 \\
\hline \multirow{4}{*}{$\begin{array}{l}\text { How did they learn about } \\
\text { this treatment? }(\mathrm{n}=93 ; 6 \\
\text { individuals did not } \\
\text { answer) }\end{array}$} & Neighbor, friend & 59 & 63.5 \\
\hline & Family & 19 & 20.4 \\
\hline & Health care personnel & 15 & 16.1 \\
\hline & Total & 93 & 100 \\
\hline
\end{tabular}

*Percentages were analyzed according to answers given. Some individuals reported using more than one me

treatment. For example, when combined with anticoagulant, anti-platelet and non-steroidal anti-inflammatory drugs, garlic has been reported to increase the risk of bleeding (Edwards et al., 2005; Hu et al., 2005). For these reasons, while taking history, health care professionals should ask patients what herbal therapies they use and should not recommend herbal therapies as a treatment for hypertension. Yoghurt with garlic, olive oil tree leaves kept in lemon juice, and laurel leaves were other herbal therapies described in this study. The subjects included in this study had been suffering from hypertension for many years and only $4.1 \%$ of them were not taking any hypertensive treatment. The most common reasons noted by the subjects for not taking hypertensive medications were finding the treatment unnecessary and unbeneficial, and being unable to afford prescriptions. According to a report issued by WHO, 17-50\% of hypertensive patients in recent studies did not comply with their treatment (WHO, 2003b). In the present study, the percentage of the patients that was incompliance with their treatment was quite high compared to studies from other countries. This may be that health care services were easily available to people in the study area and that the hypertensive patients had a health insurance. Hypertension is a chronic disease and requires continuous treatment. Lack of compliance with hypertension treatment may be attributed to the health perception of individuals. In Turkey, individuals do not consider themselves ill if they can carry out their daily routines and tasks at work. The hypertensive patient who is able to work and engage in normal activities may not see him or herself as ill, and thus he or she may think that anti-hypertensive medications are unnecessary. Health professionals play an important role in shaping/changing the health perception of individuals. When the health perception of individuals has a negative impact on their health, health professionals need to intervene to correct that view. In this study, $51.3 \%(n=99)$ of the participants 
Bahar et al., Afr J Tradit Complement Altern Med. (2013) 10(2):292-298 296 http://dx.doi.org/10.4314/ajtcam.v10i2.14

Table 2: Socio-Demographic Features of Hypertensive Patients and Herbal Therapies Practices Used by the Patients

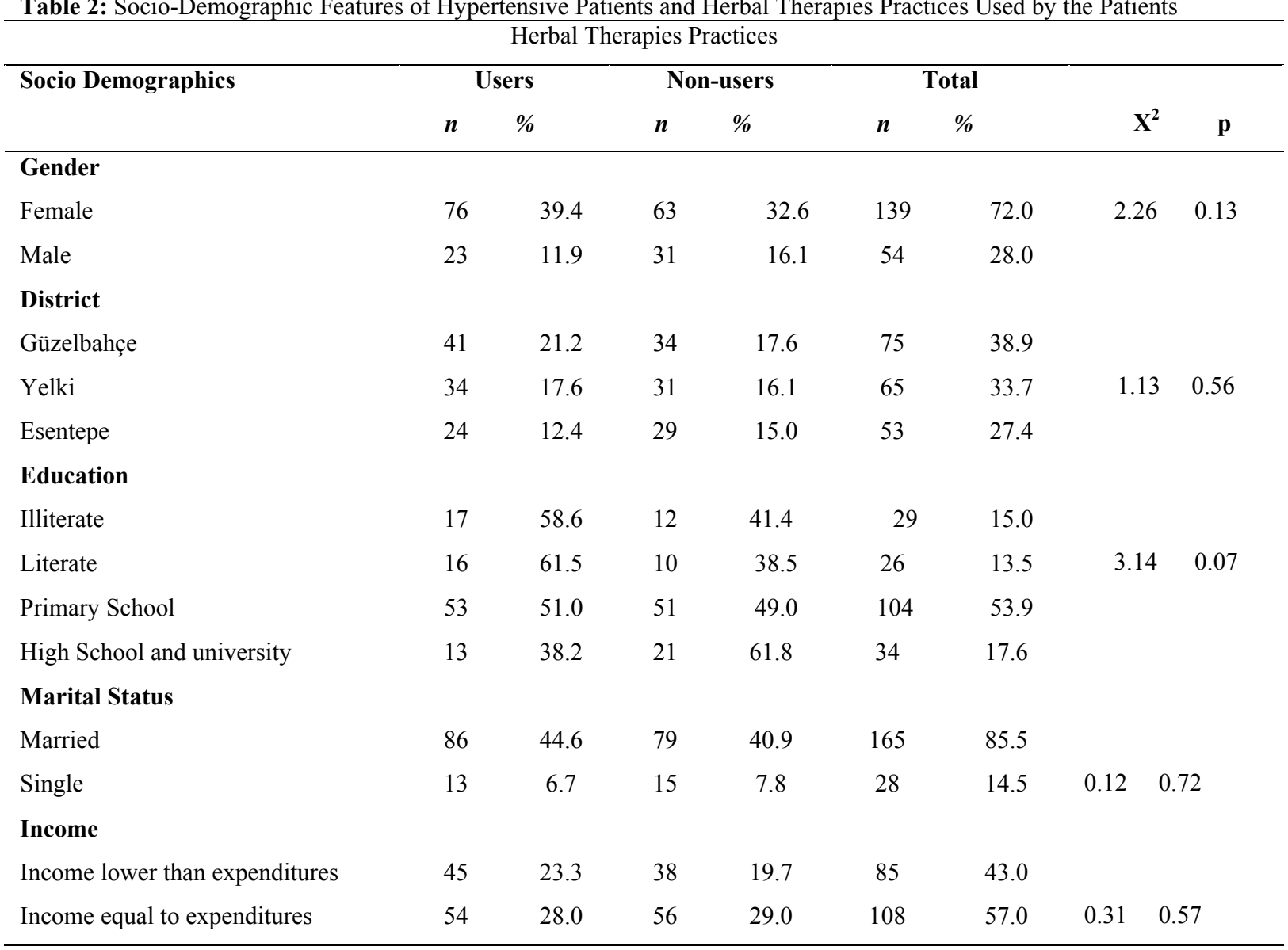

received herbal therapies and most of them were given additional medication to reduce blood pressure. Similarly, Türk and Süner (2008) found that $40.6 \%$ of the patients do not use herbal therapies, and $59.4 \%$ of the patients use herbal therapies. Among the patients that use herbal therapies, $43.3 \%$ use garlic and yogurt with garlic, and $24.6 \%$ use lemon and lemon juice to decrease the blood pressure. Adibelli, Dilek, and Akpolat (2008) stated that $72.5 \%$ of the hypertensive patients use alternative medicine and of them $40 \%$ use lemon juice to lower the blood pressure.

In contrast, Akpinar and Tezel (2003) found out that $86.9 \%$ of the hypertensive patients preferred some non-medical remedies regulating their blood pressures and $63.72 \%$ stated that these herbal therapies regulated their blood pressures. Toprak and Demir (2007) reported that $66.8 \%$ of the participants used herbal therapies to cure hypertension. We found a lower rate of hypertensive patients using herbal therapies than those reported within other studies in Turkish populations. However, the two previous studies carried out in Turkey were conduted in the eastern part of the country. The present study was conducted in Izmir -a western city in Turkey- and there are differences in cultural health behaviors between the eastern and western parts of Turkey. Herbal therapies are more frequent in the east. Among the contributory factors are: lower education levels, complete submission to societal values and traditions. Culture is one of the most important factors that affect the use of herbal therapies. The reason for the conflicting results of the studies from a country may be explained by cultural differences. Beliefs and cultural opinions can lead people to think that herbal therapies are more beneficial. Also the belief that herbal therapy is less harmful to body can cause the widespread usage of herbal therapies. Long waiting times in hospitals, expensiveness of the drugs and health perceptions can increase the utility of herbal therapies. In addition, treatment recommendations of friends, relatives and neighbors have an important role in our country. For example in a study, 33.7\% of the participants declared that they used the herbal therapies recommended by peers or neighbors (Turkish Pharmacologs Association [TPA], 2001). In this study, we think that the use of herbal therapies can be affected by social environment.

Types of herbal therapies also vary. Our study and the other studies in Turkey have suggested that lemon juice and lemon juice with garlic are the most common herbal therapies. However, unlike the two other studies from Turkey, we found that eight other plants were used either alone or in combination and that some of them were consumed as they were but some of them were boiled and the resultant water was drunk. Capraz, Dilek, and Akpolat (2007) found that garlic was frequently used to heal hypertension in the middle of Turkey. Akpınar and Tezel (2003) reported that garlic (44.3\%), lemon juice $(42.5 \%)$, airan (31\%), water boiled with olive tree leaves (23.9\%), cucumbers $(7.1 \%)$ and onion $(6.2 \%)$ were used in the east of Turkey. Khosh and Khosh (2001) reported that olive tree leaves were used to regulate blood pressure in Mediterranean countries.

In this study, most of the hypertensive subjects perceived herbal therapies to lower their blood pressure. It has been suggested in the literature that garlic, grape juice, parsley leaves and roots, green tea, flax seeds, cinnamon and yogurt, thanks to the minerals and vitamins they contain, play a role in reducing blood pressure (Castleman, 2005; Duffy et al., 1999; Fantinelli \& Mosca, 2007; Jimenez et al., 2007; Martinez, 1998; Preuss et al., 2006). However, there have been few studies 
on the effects of garlic and fifteen other types of food containing vitamin E, calcium and vitamin C; the most extensively investigated herbal therapies are garlic and policosanol (Knox \& Gaster, 2007; Preuss et al., 2006). In this study, the most frequently used herbal therapies by the hypertensive subjects were lemon juice and lemon juice with garlic. Duffy et al. (1999) found that vitamin C decreased systolic blood pressure. Chobanian et al. (2003) reported that there was no strong evidence for antihypertensive effects of garlic and recommended that hypertensive patients should be asked whether they ate garlic or not and care should be taken while using garlic as an antihypertensive remedy. It has also been reported that herbal therapies have side-effects and can be harmful when combined with drugs and therefore should not be used unless a physician approves of their use (Scott \& Elmer, 2002). In fact, garlic inhibits platelets, which causes postoperative bleeding and spontaneous epidural hematoma. When taken with anticoagulants, garlic may enhance their effects (Scott \& Elmer, 2002). The effects of these substances on blood pressure need further investigation. It is of importance to know effects of herbal therapies. As a result of an interaction with antihypertensive treatments and some kinds of food, herbal may create diuresis, hypotension and hypokalemia (Izzo, Di Carlo, Borrelli, \& Ernst, 2005; Kuhn, 2002). So it might be better to discuss the strategy to increase the patient's ability to select proper herbal therapies.

In this study, $63.5 \%$ of the hypertensive subjects who used herbal therapies learned about the therapies from their neighbors. Similarly, Akpinar and Tezel (2003) reported that $69.03 \%$ of the hypertensive patients learned herbal therapies from their neighbors, $16.81 \%$ from their families, $8.85 \%$ from the media and $5.31 \%$ from health professionals. In contrast, Engin and Pasinlioğlu (2002) found that only $27.4 \%$ of hypertensive patients reported neighbors as the source of information about herbal therapies. Social and cultural factors play an important role in health behaviors. One of the most important features of Turkish culture is to share things, ideas, experiences with others. When individuals receive a benefit from a particular method, they share it with their close friends and relatives. In fact, we found that hypertensive patients shared their experiences regarding herbal therapies with their friends, family members and neighbors. However, sharing such experiences may harm some people. In fact, the interactions of herbal therapies with drugs may have negative effects and the mode of action is not clear in herbal therapies. In this study, there were no statistically significant differences between the tendency to use herbal therapies and gender, education, marital status, having health insurance, income or place of residence (district) among the hypertensive subjects. However, differences based on education almost reached a level of significance. Herbal therapies tended to be used by females and by those who had not received education through the Ministry of Education programs. It may be that the percentage of the subjects who used herbal therapies was nearly the same as those who did not although they differed in gender, education, place of residence, marital status and income. This might be the reason for lack of a significant difference between the use of herbal therapies and the independent variables. The results of this study contradicted with previous research. A study showed that as education level increases, herbal therapies usage rate decreases (TPA, 2001). Similarly, Buettner et al. (2007) reported that herbal therapies usage increases as education level increases.

\section{Limitations}

Limitation of this study is that we couldn't determine other alternative methods used by other hypertensive patients but reported only the herbal therapies administered to our study group.

\section{Recommendations for Further Research}

Randomized, controlled studies are needed to determine the effects of herbal therapies on hypertension. Also, the effect of reaching the health services providing herbal therapies can be a research topic.

\section{Implications for Nursing Practice}

Health professionals who offer the first-line health services, especially nurses, should follow patients regularly and provide training for anti-hypertensive treatment such as healthy nutrition, exercise, drug use and the importance of regular blood pressure monitoring. It is important to provide patient with evidence based data regarding their use. They should also know what herbal therapies are used and how they affect patients, and should inform patients about the effects of herbal therapies. During home visits nurses must plan training programs for the hypertensive patients that use herbal therapies which might have negative effects on their health.

\section{References}

1. Adibelli, Z., Dilek, M., \& Akpolat, T. (2008). Lemon juice as an alternative therapy in hypertension in Turkey. International Journal of Cardiology, 30.

2. Akpınar, R. B., \& Tezel, A. (2003). Natural methods used for reduction of blood pressure. Atatürk University School of Nursing Journal, 6(2), 34-39.

3. Al-Qattan, K. K., Thomson, M., Al-Mutawa'a, S., Al-Hajeri, D., Drobiova, H., \& Ali, M. (2006). Nitric oxide mediates the bloodpressure lowering effect of garlic in the rat two-kidney, one-clip model of hypertension. Journal of Nutrition, 136, 774-776.

4. Buettner, C., Phillips, R. S., Davis, R. B., Gardiner, P., \& Mittleman, M. A. (2007). Use of dietary supplements among United States adults with coronary artery disease and atherosclerotic risks. American Journal of Cardiology, 99, 661-666.

5. Castleman, M. (2005). Powerful parsley: Promote this garnish to salad centerpiece for clean breath, a settled stomach, and a big hit of antioxidants. Natural Health. Available at: http://findarticles.com/p/articles/mi_m0NAH/is_1_36/ai_n15895453 (Accessed 15 May 2011). 
6. Chobanian, A.V., Bakris, G. L., Black, H. R., Cushman, W. C., Green, L. A., Izzo, J. L., et al. (2003). Seventh report of the Joint National Committee on prevention, detection, evaluation and treatment of high blood pressure. Hypertension, 42(6), $1206-1252$.

7. Capraz, M., Dilek, M., \& Akpolat, T. (2007). Garlic, hypertension and patient education. International Journal of Cardiology, 121(1), 130-131.

8. Dharmananda, S. (2003). Safety issues affecting herbs: Herbs that may increase blood pressure. Available at: http://www.itmonline.org/arts/hypertension.htm (accessed 15 May 2011).

9. Dosh, S. A. (2002). The treatment of adults with essential hypertension. The Journal of Family Practice, 51(1), 74-80.

10. Duffy, S. J., Gokce, N., Holbrook, M., Huang, A., Frei, B., Keaney, J. F., et al. (1999). Treatment of hypertension with ascorbic acid. Lancet, 354, (9195), 2048-2049.

11. Edwards, Q. T., Colquist, S., \& Maradiegue, A. (2005). What's cooking with garlic: Is this complementary and alternative medicine for hypertension? Journal of American Academy of Nurse Practitioners, 17(9), 381-385.

12. Engin, R., \& Pasinlioğlu, T. (2002). Beliefs and traditional practices about infertility among women from Erzurum. Atatürk University School of Nursing Journal, 5(1), 34-39.

13. Fantinelli, J., \& Mosca, S. M. (2007). Cardioprotective effects of a 393 non-alcoholic extract of red wine during ischaemia and reperfusion in spontaneously hypertensive. Clinical \& Experimental Pharmacology \& Physiology, 34(3), 166-169.

14. Fields, L. E., Burt V. L., Cutler, J. A., Hughes, J., Roccella, E. J., \& Sorlie, P. (2004). The burden of adult hypertension in the United States 1999 to 2000: a rising tide. Hypertension, 44(4), 398-404.

15. Goertz, C. H., Grimm, R. H., Svendsen, K., \& Grandits, G. (2002). Treatment of hypertension with alternative therapies study: A randomized clinical treatment. Journal of Hypertension, 20(10), 2063-2068.

16. He, F. J., \& MacGregor, G. A. (2002). Effect of modest salt reduction on blood pressure: A meta-analysis of randomized trials implications for public health. Journal Hum Hypertension, 16(11), 761- 770.

17. Hu, Z., Yang, X., Ho, P. C., Chan, S. Y., Heng, P. W., \& Chan, E. (2005). Herb-drug interactions: a literature review. Drugs, 65(9), 1239-1282.

18. Izzo, A. A., Di Carlo, G., Borrelli, F., \& Ernst, E. (2005). Cardiovascular pharmacotherapy and herbal medicines: the risk of drug interaction. International Journal of Cardiology, 98(1), 1-14.

19. Jiménez, R., López-Sepúlveda, R., Kadmiri, M., Romero, M., Vera, R., \& Sánchez, M., et al. (2007). Polyphenols restore endothelial function in DOCA-salt hypertension: role of endothelin-1 and NADPH oxidase. Free Radical Biology \& Medicine, 43(3), 462-473.

20. Khosh, F., \& Khosh, M. (2001). Natural approach to hypertension. Alternative Medicine Review, 6(6),590-600.

21. Kirkpantur, A., \& Turgan, Ç. (2006). Hypertension of aged groups. Geriatr and Gerontoloji, 805-818.

22. Knox, J., \& Gaster, B. (2007) Dietary supplements for the prevention and treatment of coronary artery disease. Journal of Alternative Complementary Medicine, 13(1), 83-95.

23. Kuhn, M. A. (2002). Herbal remedies: drug-herb interactions. Critical Care Nurse, 22(2), 22-32.

24. Mansoor, G. A. (2001). Herbs and alternative therapies in the hypertension clinic. American Journal of Hypertension, 14, 971975.

25. Martinez, C. (1998). Calcium and hypertension. Nutrition Bytes, 4(2), 4.

26. Nahas, R. (2008). Complementary and alternative medicine approaches to blood pressure reduction. Prectica Medicala, 3, 185190.

27. National Heart Lung and Blood Institute. (2008) Who Is At Risk for High Blood Pressure? Retrieved April 20, 2011, from http://www.nhlbi.nih.gov/health/dci/Diseases/Hbp/HBP_WhoIsAtRisk.html

28. Preuss, H. G., Echard, B., Polansky, M. M., \& Anderson, R. (2006). Whole cinnamon and aqueous extracts ameliorate sucroseinduced blood pressure elevations in spontaneously hypertensive rats. Journal of American College Nutrition, 25(2), $144-150$.

29. Scott, G. N., \& Elmer, G. W. (2002). Update on natural product--drug interactions. American Journal of Health System Pharmacy, 59(4), 339-347.

30. Taddei, S., Virdis, A., Ghiadoni, L., Magagna, A., \& Salvetti, A. (1998). Vitamin C improves endothelium-dependent vasodilation by restoring nitric oxide activity in essential hypertension. Circulation, 97(22), 2222-2229.

31. Toprak, D., \& Demir, S. (2007). Treatment choices of hypertensive patients in Turkey. Behavioral Medicine, 33, 5-10.

32. Türk, N., \& Süner, A. (2008). Non-pharmacological method and the analysis of antihypertension medication. Göztepe Medicine Journal, 23(4):133-142.

33. Turkish Pharmacologs Association Report (2001). Compliance with treatment of patients with hypertension and antihypertensive drug use characteristics of the patients in Turkey. Retrieved May 3, 2011, from http://www.ekutuphane.teb.org.tr/pdf/raporlar/tc_hipertansiyon/10.pdf

34. Vasan, R. S., Beiser, A., Seshadri, S., Larson, M. G., Kannel, W. B., D'Agostino, R. B., et al. (2002). Residual lifetime risk for developing hypertension in middle-aged women and men: The Framingham Heart Study. Journal of the American Medical Association, 287(8), 1003-1010.

35. Wangzhong, Q. (1996). One medicine, two cultures evaluation of hypertension 492 treatment by traditional Chinese medicine. Retrieved May 16, 2011, from http://www.gfmer.ch/TMCAM/Hypertension/Evaluation_hypertension_treatment_Traditional_Chinese_Medicine.htm

36. World Health Organization. (2005a). Pharmacy-Based Hypertension Management Model: Protocol and Guidelines. A joint CINDI/EuroPharm Forum Project. Retrieved May 18, 2011, from https://files.pbworks.com/download/PI0Bzm3e6R/europharm/19341776/hypertension.pdf

37. World-Health-Organization. (2003b) Adherence to Long Term Therapy: Evidence for Action. Retrieved May 16, 2011, from http://apps.who.int/medicinedocs/pdf/s4883e/s4883e.pdf. 